\title{
Structural, optical, and luminescence properties of ZnO:Ga optical scintillation ceramic
}

\author{
E. I. Gorokhova, ${ }^{1,7}$ S. B. Eron'ko, ${ }^{1}$ E. A. Oreshchenko, ${ }^{1}$ A. V. Sandulenko, ${ }^{1}$ P. A. Rodny ${ }^{2},{ }^{2}$ \\ K. A. Chernenko, ${ }^{2,3}$ I. D. Venevtsev, ${ }^{2}$ A. M. Kul'kov, ${ }^{4}$ Faina Muktepavela, ${ }^{5}$ and Plamen Boutachkov ${ }^{6}$ \\ ${ }^{1}$ S. I. Vavilov State Optical Institute Scientific Production Enterprise, St. Petersburg, Russia \\ ${ }^{2}$ Peter the Great St. Petersburg Polytechnic University, St. Petersburg, Russia \\ ${ }^{3}$ MAX IV Laboratory, Lund University, P.O. Box 118, SE-22100 Lund, Sweden \\ ${ }^{4}$ St. Petersburg State University, St. Petersburg, Russia \\ ${ }^{5}$ Institute of Solid State Physics, University of Latvia, Riga, Latvia \\ ${ }^{6} \mathrm{GSI}$ Helmholtzzentrum für Schwerionenforschung GmbH, Darmstadt, Germany \\ ${ }^{7}$ e-mail: E.Gorokhova@rambler.ru
}

\begin{abstract}
This paper discusses the characteristics of $\mathrm{ZnO}$ and $\mathrm{ZnO}: \mathrm{Ga}$ ceramics fabricated by uniaxial hot pressing. The short-wavelength transmission limit of zinc oxide ceramics is in the 370-nm region; the long-wavelength limit is determined by the free-charge-carrier concentration and lies in the interval from 5 to $9 \mu \mathrm{m}$. The total transmittance of such ceramics in the visible and near-IR regions is about $70 \%$ when the sample is $0.5 \mathrm{~mm}$ thick. The luminescence spectrum is represented by a broad emission band with maximum at $580 \mathrm{~nm}$, having a defect nature. The introduction of 0.03-0.1 mass \% gallium into the zinc oxide structure inhibits grain growth and increases the free-charge-carrier concentration to $3.44 \times 10^{19} \mathrm{~cm}^{-3}$. As the gallium concentration increases in the range 0.05-0.1 mass \% in a ceramic of composition $\mathrm{ZnO}: \mathrm{Ga}$, the defect luminescence band is suppressed and a characteristic exciton luminescence is formed with a maximum corresponding to $389 \mathrm{~nm}$ and a damping time constant of $1.1 \mathrm{~ns}$.
\end{abstract}

Zinc oxide $\mathrm{ZnO}$ is a multifunctional material with interesting characteristics that has been widely used for several decades in the most varied realms [1]. It is a direct semiconductor that is $n$-type in the undoped form and has a wide, 3.37-eV band gap at room temperature. The excitons in $\mathrm{ZnO}$ possess the large binding energy of $60 \mathrm{meV}$, which gives them high temperature stability (up to $350 \mathrm{~K}$ ).

Two emission bands are recorded in the various forms of $\mathrm{ZnO}$ : a short-wavelength band close to the crystal's absorption edge, the so-called edge luminescence, which has an exciton nature, and a broad long-wavelength luminescence (LWL) band, which has a defect origin. The LWL maximum is usually found in the 510-600-nm region. The fall-off (damping) constant of the edge luminescence is about $1 \mathrm{~ns}$, and the characteristic luminescence time of the LWL is in the range from a few to several hundred microseconds [2]. The introduction of dopants ( $\mathrm{Ga}, \mathrm{In}$, or $\mathrm{Al}$ ) into the $\mathrm{ZnO}$ creates donor centers, and this increases the intensity of the excitonic luminescence and reduces the intensity of the defect band. The introduction of trivalent impurities not only reduces the intensity of the defect luminescence but also shortens its damping time. Studies have been carried out of how $\mathrm{Ga}$ affects the characteristics of $\mathrm{ZnO}$ powders [3], films [2,4], single crystals [5], and ceramics $[6,7,8]$. Ceramic $\mathrm{ZnO}: \mathrm{Ga}$ is of special interest for its use in scintillation detectors, since it possesses a short damping time of the exciton luminescence (less than one nanosecond) and high radiation stability and is a comparatively inexpensive material. A $\mathrm{ZnO}$ :Ga scintillator in the form of films is used as an efficient $\alpha$-particle detector in deuterium/tritium neutron generators [4].

\section{EXPERIMENTAL TECHNIQUE}

Russian-made commercial $\mathrm{ZnO}$ powder (TU-6-09-2175-77) (Technical Specification) (99.5\%, VHP 14-2, p. 4) and powder from Sigma Aldrich, USA (99.999\%, Bath 3 MKBC3549) were used to obtain vhp-grade ceramic.

The gallium dopant was introduced in the form of its oxide (Alfa Aesar, USA, commercial grade Puratronic, 99.999\%) by mechanically mixing it with $\mathrm{ZnO}$. The gallium concentration was varied in the range from 0.03 to 0.1 mass \%. The ceramic samples were fabricated in the form of disks and after mechanical processing were $24 \mathrm{~mm}$ in diameter 
Table 1. Concentration of Ga Dopant Introduced into the Samples

\begin{tabular}{lcc}
\hline Sample No. & $\begin{array}{c}\text { Concentration of } \\
\text { Ga, mass \% }\end{array}$ & $\begin{array}{c}\text { Manufacturer of } \\
\text { ZnO powder }\end{array}$ \\
\hline 1a & 0 & Russia, TU-6-09-2175-77 \\
2a & 0.03 & Russia, TU-6-09-2175-77 \\
3a & 0.05 & Russia, TU-6-09-2175-77 \\
4a & 0.075 & Russia, TU-6-09-2175-77 \\
5a & 0.1 & Russia, TU-6-09-2175-77 \\
1b & 0 & USA, Sigma Aldrich \\
3b & 0.05 & USA, Sigma Aldrich \\
4b & 0.075 & USA, Sigma Aldrich \\
5b & 0.1 & USA, Sigma Aldrich \\
\hline
\end{tabular}

and $0.45-0.5 \mathrm{~mm}$ thick. Data on the composition of the samples are presented in Table 1.

X-ray structural studies of the ceramic were carried out on a DRON-2 X-ray diffractometer with a copper anode and a nickel filter. The results were interpreted on the basis of the data of the JCPDS (Joint Committee on Powder Diffraction Standards) cards, card number 36-1451. The crystal-lattice parameters were determined on the basis of the system of (105) and (300) crystallographic planes with large reflection angles $2 \theta\left(104.12\right.$ and $\left.110.52^{\circ}\right)$. The predominant orientation of the grains in the ceramic samples was estimated using the texture factor, calculated from [9]

$$
T_{c}(b k l)=\frac{\frac{I(b k l)}{I_{0}(b k l)}}{\left(\frac{1}{N}\right)\left[\sum_{N} \frac{I(b k l)}{I_{0}(b k l)}\right]},
$$

where $T_{c}(h k l)$ is the texture factor of the $(b k l)$ plane, $I$ is the measured intensity of the corresponding peak, $I_{0}$ is the standard intensity according to JCPDS, and $N$ is the number of diffraction peaks. It follows from this equation that $T_{c}>1$ when the corresponding $(h k l)$ plane has a predominant orientation, and $T_{c}<1$ in randomly oriented grains.

The morphological composition of the powders was studied and the particle size was estimated by means of the Zeiss EVO $50 \mathrm{XVP}$ scanning electron microscope and the TecnaiGF20 transmission electron microscope. The POLAM P-312 optical microscope was used to investigate the microstructure of the ceramic samples. The total transmittance was measured by means of a Lambda 1050 spectrometer made by PerkinElmer, equipped with an integrating-sphere attachment, and the spectral transmittance and reflectance in the IR were measured by means of an FSM Fourier spectrometer. The luminescence spectra were measured with continuous $\mathrm{X}$-ray excitation (the anode voltage of the X-ray tube was $40 \mathrm{kV}$ ) in transmitted and reflected light. The X-ray luminescence spectra of the samples were recorded using an MDR-3 monochromator with gratings having line density 1200 or $600 \mathrm{~mm}^{-1}$. An R928 broad-band photomultiplier with a Hamamatsu pre-amp was used as the radiation detector, after which the signal was digitized by a 16-bit National Instruments analog-to-digital converter. The resulting spectra were corrected, using the transmittance of the monochromator and the spectral sensitivity of the photomultiplier. Two original experimental devices were used to measure the luminescence-damping curves: the damping of the defect luminescence band of undoped ceramics was measured in the range from hundreds of nanoseconds to a few milliseconds by means of an X-ray source that made it possible to obtain pulses from 100 to 2000 ns wide, described in detail in [10]; the damping of the excitonic luminescence band of the gallium-doped ceramics was measured in the range from a few to hundreds of nanoseconds by means of an X-ray source with a pulse width less than $1 \mathrm{~ns}$, described in [11].

\section{RESULTS AND DISCUSSION}

It was established from the X-ray structural analysis that all the ceramic samples have the same hexagonal phase of $\mathrm{ZnO}$.

Figures 1(a) and 1(b) show how the lattice parameters of the ceramic samples fabricated from the TU-6-09-2175-77 and Sigma Aldrich $\mathrm{ZnO}$ powders depend on the dopant concentration. As can be seen from the figure, parameter $a$ is virtually independent of the amount of $\mathrm{Ga}$ and oscillates within the limits from 3.2519 to $3.2527 \AA$. The presence of the dopant in the $\mathrm{ZnO}$ lattice is tracked using the character of the change of parameter $c$. The introduction of an amount of gallium from 0.03 to 0.075 mass \% causes its value to decrease. The most substantial decrease of the parameter in this case, from 5.2071 to $5.2032 \AA$, is observed at the minimum concentration of Ga: 0.03 mass \% [Fig. 1(a)]. As the concentration is increased from 0.075 to 0.1 mass $\%$, parameter $c$ tends to increase, but only to a value comparable with the parameter of the undoped $\mathrm{ZnO}$ ceramic.

The ambiguous (nonmonotonic) character of the dependence of parameter $c$ is possibly explained by the incorporation of $\mathrm{Ga}$ into various crystal-lattice sites of $\mathrm{ZnO}$. Gallium can occupy regular positions of the biotite structure, replacing zinc ions with tetrahedral coordination, or can be incorporated into octahedral interstices. This is mentioned, in particular, in papers devoted to the study of $\mathrm{ZnO}: \mathrm{Ga}$ films $[12,13]$.

Since the gallium in the oxide $\mathrm{G}_{2} \mathrm{O}_{3}$, in the form of which the dopant was introduced, has octahedral coordination, such a position can be even more preferred under certain conditions. Since the effective ionic radius of $\mathrm{Ga}^{3+}$ both with tetrahedral coordination $(0.47 \AA)$ and with octahedral coordination $(0.62 \AA)$ is less than that of $Z^{2+}(0.62$ and $0.74 \AA$, respectively [14]), parameter $c$ must decrease, and this is what is observed.

The results of the study of how the activator concentration affects the texture factor $T_{c}$ of the ceramic samples are shown in Figs. 1(c) and 1(d). All of these samples are characterized by predominant orientation of the grains along the (100) and (110) planes of the prism and weak texturing along the (101) plane of the rhombohedron. The presence of gallium in the $\mathrm{ZnO}$ structure results in a gradual reduction of $T_{c}$ (100) and $T_{c}(110)$ as its concentration increases and has virtually no effect on $T_{c}$ of the (101) plane of the rhombohedron but manifests a definite tendency to prefer to orient the grains along the (102) plane of the rhombohedron in the ceramic fabricated from Sigma Aldrich powder.

Figure 2 shows electron micrographs of the starting powders and the microstructure of the ceramic samples fabricated from TU-6-09-2175-77 $\mathrm{ZnO}$ powders produced in Russia [Fig. 2(a)] and produced by Sigma Aldrich [Fig. 2(b)], along 
(a)

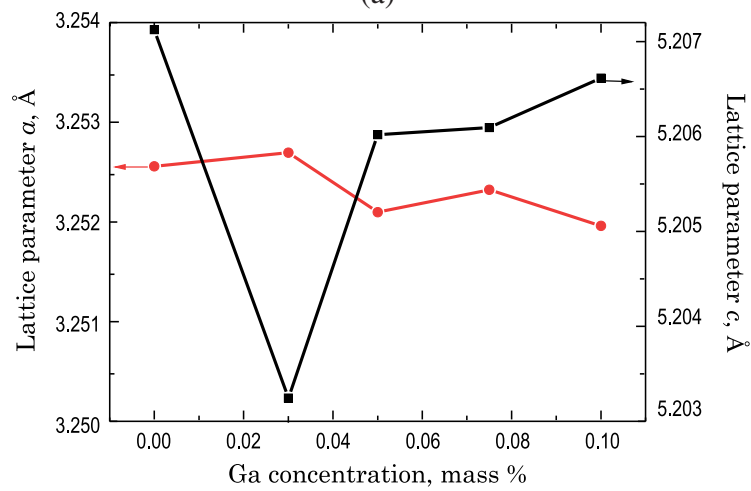

(c)

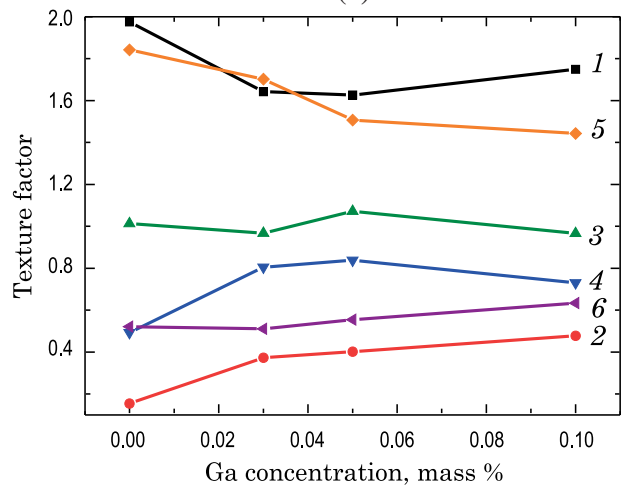

(b)

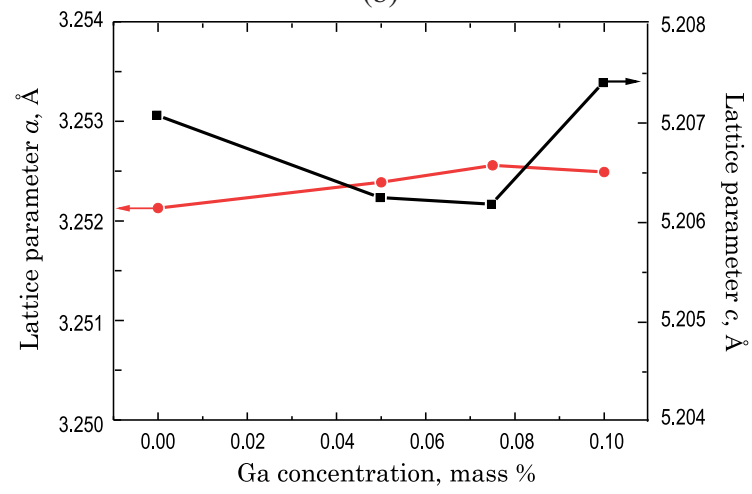

(d)

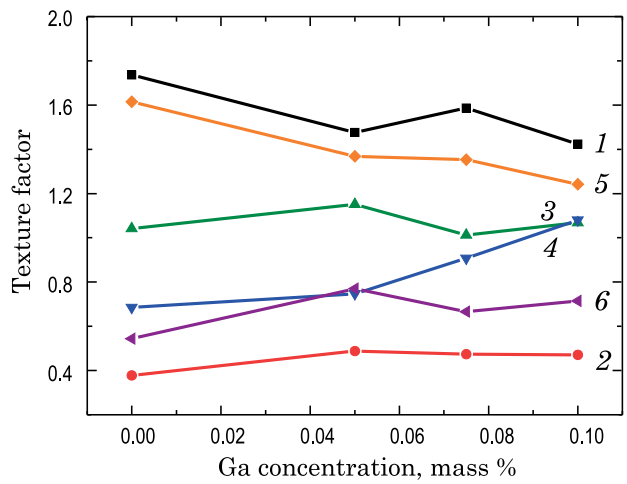

Fig. 1. The effect of a Ga impurity on the (a), (b) lattice parameters and (c), (d) texture factors of ceramics fabricated from $\mathrm{ZnO}$ using (a), (c) TU-6-09-2175-77 and (b), (d) Sigma Aldrich powders. The curves in graphs (c) and (d) correspond to the following planes: 1-(100), 2-(002), 3-(101), 4-(102), 5-(110), 6-(103).

with histograms displaying the size distribution of the grains in these samples.

The morphological content of the TU-6-09-2175-77 $\mathrm{ZnO}$ powder made in Russia is represented by acicular and elongated varieties of nanorod grains of size $(50-70) \times 150 \mathrm{~nm}$ and $45 \times 500 \mathrm{~nm}$. The predominant habitus of these grains is hexagonal-prismatic. Moreover, large grains are present, frequently with faceting, with a size from $200 \times 750 \mathrm{~nm}$ to $1 \mu \mathrm{m}$ and various agglomeration formations.

Crystal grains with a size of $50 \times 100,100 \times 200$, and $100 \times$ $500 \mathrm{~nm}$ are also present in the Sigma Aldrich $\mathrm{ZnO}$. However, unlike the TU-6-09-2175-77 powder made in Russia, this powder, along with nanorod grains, contains flattened tabletlike grains having a tetrahedral-prismatic habitus. Moreover, aggregates in the form of chains and rings of small particles are present in the powder. It can thus be stated that both starting powders agglomerate to a significant degree.

The microstructure of the two undoped ceramics is similar and is represented by grains that have a shape close to isometric, with sizes in the range from 10 to $35 \mu \mathrm{m}$, and this significantly exceeds the particle size of the starting powder as a consequence of intensive recrystallization processes.

The presence of the gallium dopant changes the character of the microstructure of the $\mathrm{ZnO}$ ceramic, although not so radically as is observed when it is doped with indium [15]. Subdivision and change of the morphology of the grains occurs.
The reduction of the grain size shows up most strongly when the minimum amount of gallium in the chosen range is introduced into the composition of the starting powder. The microstructure of this ceramic (Table 1, sample 2a) is made up of grains whose size lies in the interval from 5 to $15 \mu \mathrm{m}$. A further increase in the gallium concentration results in the appearance, along with the small grains, of larger ones with a size of up to 20-25 $\mu \mathrm{m}$, the number of which predominates in samples fabricated from TU-6-09-2175-77 powder. As the gallium concentration runs from 0.075 to 0.1 mass \% (samples $4 \mathrm{~b}$ and $5 \mathrm{~b}$, Table 1) in the ceramic samples fabricated from the Sigma Aldrich powder, grains of indefinite shape appear with wavy boundaries having no distinct faceting. Nevertheless, grains of another shape [inset to Fig. 2(b)] are also observed in sample $5 \mathrm{~b}$ (Table 1); these are more isometric, like that which characterizes the ceramic with an analogous concentration of gallium (Table 1, 5a).

The resulting data show the complex character of the effect of the gallium impurity on the recrystallization processes that occur in $\mathrm{ZnO}$ under conditions of uniaxial hot pressing, as well as making a definite contribution of special properties of the starting powders to the microstructure of the ceramics. Further, more intensive study is required for the interpretation of these results; nevertheless, it is possible to make certain assumptions.

The changes that occur can be explained by the fact that, as is well known, small concentrations of impurities in most cases 
(a)

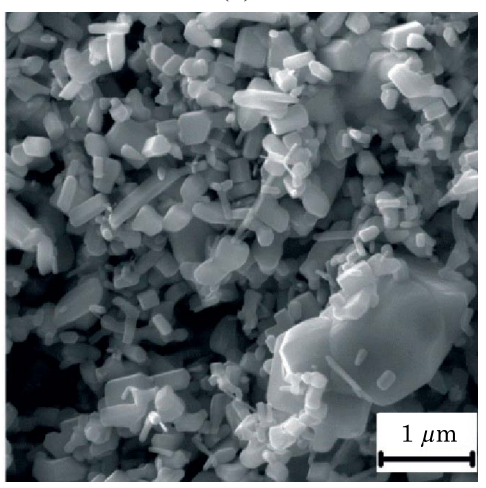

(d)

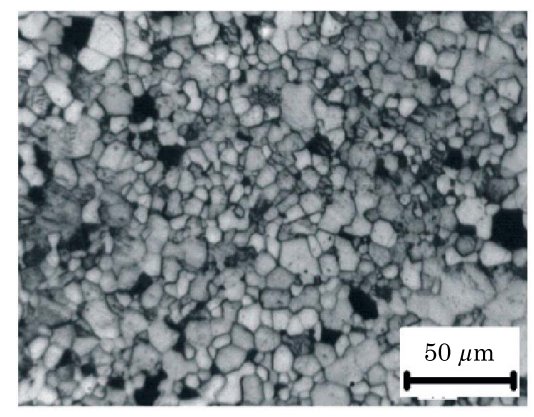

(g)

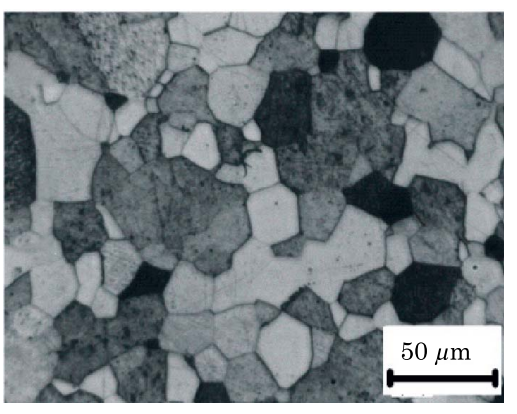

(j)

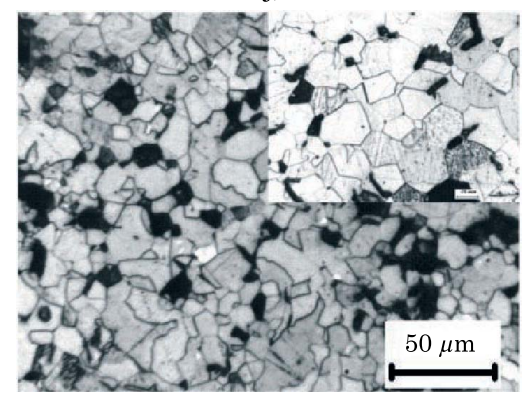

(b)

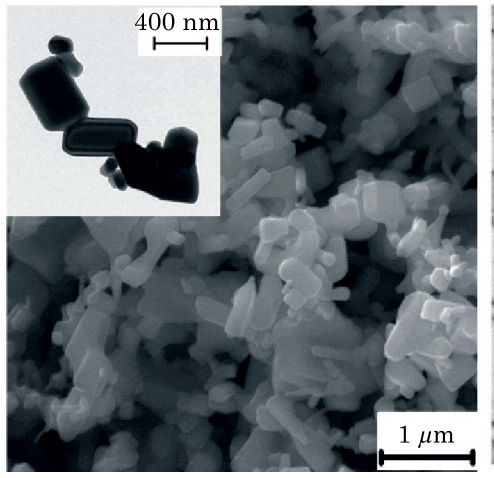

(e)

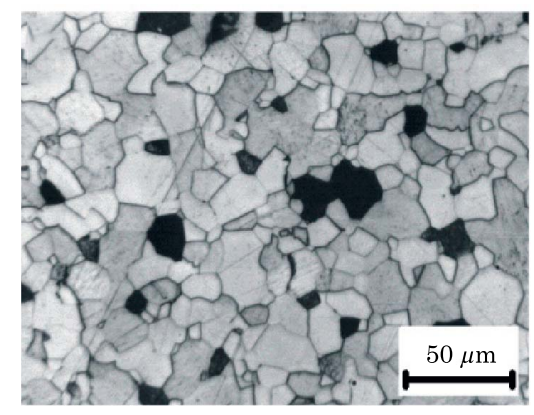

(h)

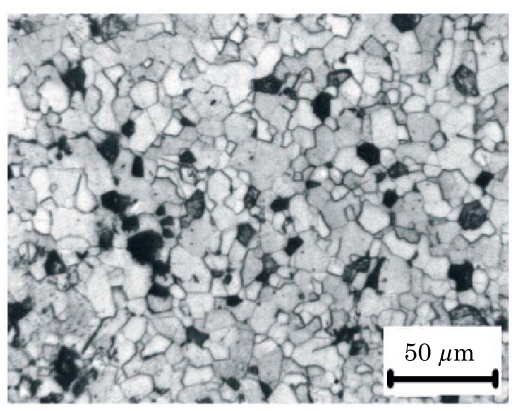

(c)

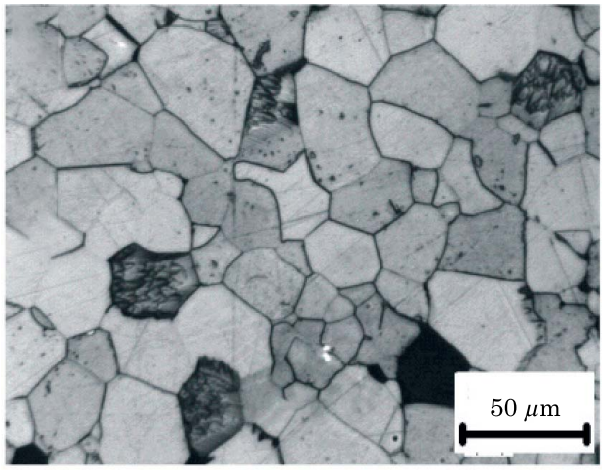

(f)

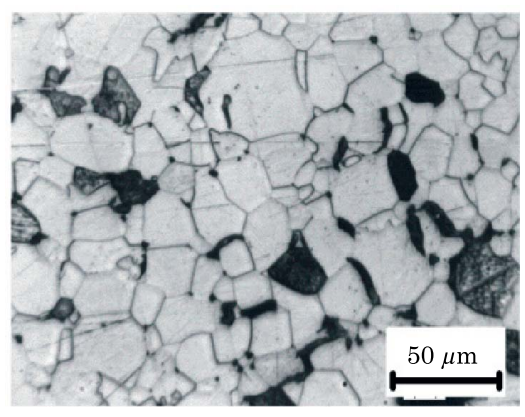

(i)

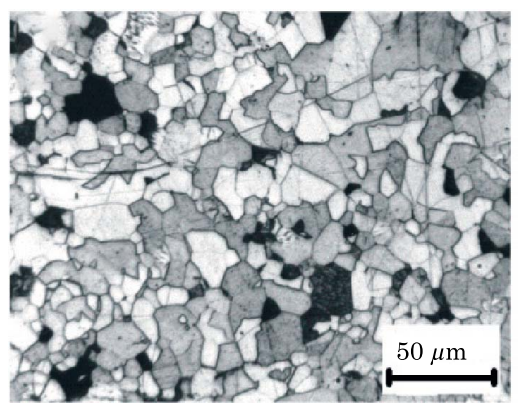

(k)

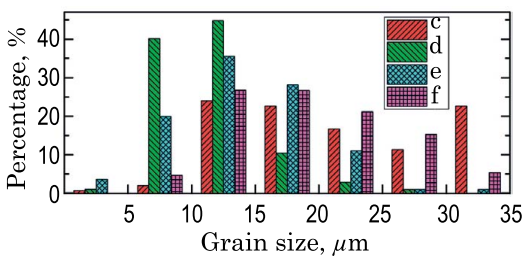

(1)

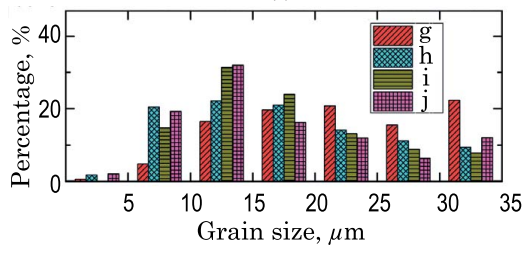

Fig. 2. Micrographs of the (a) TU-6-09-2175-77 and (b) Sigma Aldrich $\mathrm{ZnO}$ starting powders used as a basis for fabricating ZnO ceramic samples (c-sample 1a, d-2a, e-3a, and f-5a, Table 1) and $\mathrm{ZnO}: \mathrm{Ga}$ samples (g-sample 1b, h-3b, i-4b, and j-5b, Table 1), and the distribution over grain sizes of the (k) $\mathrm{ZnO}$ and (l) $\mathrm{ZnO}: \mathrm{Ga}$ samples.

raise the recrystallization-onset temperature [16]. They retard the recrystallization as a consequence of their elastic and possibly chemical interactions with dislocations and grain boundaries. It has been established that this change is most abrupt when low-solubility additives are incorporated in another type of lattice and when the matrix ions and impurity ions greatly differ in size. Moreover, the retardation becomes less efficient when the radius of the impurity ion is less than that of the 
(1a)

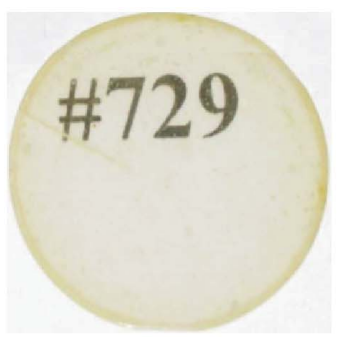

(1b)

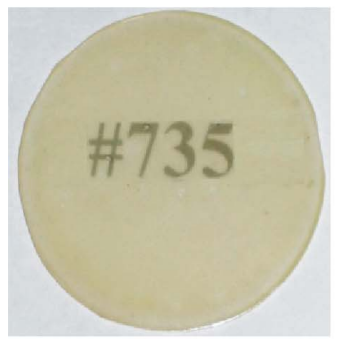

(2a)

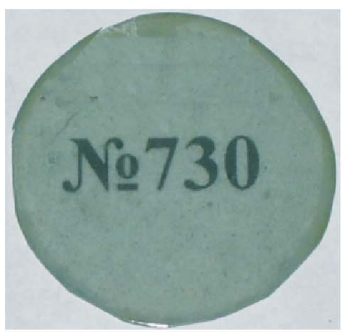

(3b)

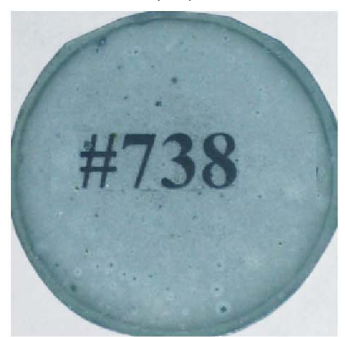

(3a)

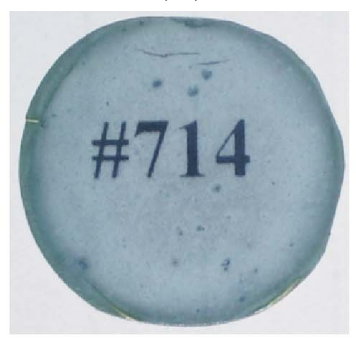

(4b)

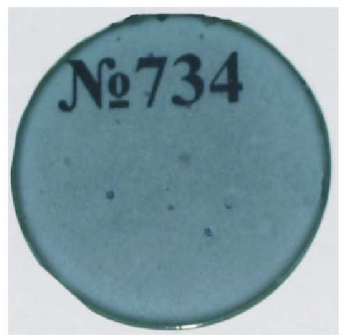

(5a)

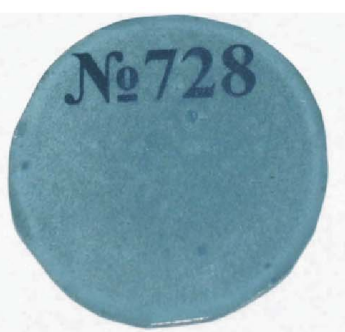

(5b)

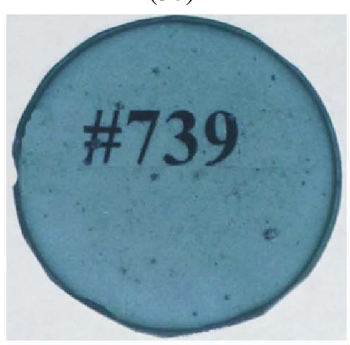

Fig. 3. Typical photographs of $\mathrm{ZnO}$ and $\mathrm{ZnO}$ :Ga samples fabricated from TU-6-09-2175-77 (1a, 2a, 3a, 5a) and Sigma Aldrich (1b, 3b, 4b, 5b) powders. The numbers in the photographs correspond to the sample numbers given in Table 1.

matrix ion. In our case, the solubility of $\mathrm{Ga}$ in $\mathrm{ZnO}$ according to various estimates is $0.5-1.0$ at $\%$, and this characterizes three-dimensional structures produced by solid-state synthesis [17]. Unlike the hexagonal structure of $\mathrm{ZnO}$, the oxide of the introduced gallium impurity $\left(\mathrm{Ga}_{2} \mathrm{O}_{3}\right)$ has a monoclinic structure; finally, the ionic radius of $\mathrm{Ga}^{3+}$ is less than that of $\mathrm{Zn}^{2+}$.

The data on nanocrystalline powders and films of $\mathrm{ZnO}: \mathrm{Ga}$ obtained by different methods also show that the gallium impurity has a substantial effect on the microstructure of $\mathrm{ZnO}$. In most cases, the size of the crystals is observed to decrease as the dopant concentration increases [17].

Characteristic photographs of $\mathrm{ZnO}$ and $\mathrm{ZnO}$ :Ga ceramics fabricated from TU-6-09-2175-77 and Sigma Aldrich powders are shown in Fig. 3. The samples of undoped $\mathrm{ZnO}$ ceramic are colored light yellow. When gallium is introduced into the starting powder, the ceramic acquires a sky-blue coloration whose intensity increases as the concentration increases. In this series, a greenish tint is observed only in the sample that contains 0.03 mass $\%$ of gallium (Table 1, 2a). Inhomogeneities are noted in the samples in some photographs, and this results from agglomeration of the starting powders.

Figure 4 shows the total transmission spectra (from the nearUV to the near-IR) of ceramic samples fabricated from the vhpgrade TU-6-09-2175-77 and Sigma Aldrich powders [Figs. 4(a) and $4(\mathrm{~b})]$ and the transmission and reflection spectra in the mid-IR range of $\mathrm{ZnO}$ and $\mathrm{ZnO}: \mathrm{Ga}$ ceramics [Figs. 4(c) and 4(d)]. The short-wavelength transmission limit of the undoped ceramics (samples 1a, 1b, Table 1) lies in the 370-nm region, but the total transmittance in the visible and near-IR is of the order of 65\%-70\% [Figs. 4(a) and 4(b)]. For polycrystalline hexagonal material with predominant orientation of the grains along the planes of the prism, this is a fairly high level, approaching the transmittance of single-crystal $\mathrm{ZnO}$ [18]. Certain differences in the character of the transmittance curves of samples $1 \mathrm{a}$ and $1 \mathrm{~b}$ (Table 1) are observed in the near-IR:
The total transmittance of the $\mathrm{ZnO}$ ceramic fabricated from the Sigma Aldrich powder (Table 1, 1b) continuously increases in the range under consideration, unlike the $\mathrm{ZnO}$ ceramic fabricated from the TU-6-09-2175-77 powder (Table 1, 1a), in which a gradual decrease in the transmittance is observed in the region beyond $870 \mathrm{~nm}$. The differences in the transmittance of these ceramics are more substantial in the mid-IR, as can be seen in Fig. 4(c). Ceramic 1b has high transmittance, while the long-wavelength limit of the transmittance is located in the $9-\mu \mathrm{m}$ region, unlike ceramic $1 \mathrm{a}$, the corresponding limit of which lies in the $5-\mu \mathrm{m}$ region. This can result from the higher concentration of free charge carriers in ceramic la by comparison with ceramic $1 \mathrm{~b}$. Studies of the IR transmittance and reflectance carried out on $\mathrm{ZnO}$ single crystals show that characteristic free-carrier absorption is observed in the 3-10- $\mu \mathrm{m}$ region, which changes to lattice self-absorption in the $9-35-\mu \mathrm{m}$ range [19]. Additional studies are required to explain why absorption appears at 3.43 and $3.51 \mu \mathrm{m}$ in both undoped ceramics. Nevertheless, based on the data of [20], it can be assumed that these peaks are associated with $\mathrm{C}-\mathrm{H}$ vibrations. It was pointed out by the authors of [20] that such bands are often observed in the spectra of $\mathrm{ZnO}$ single crystals, films, and nanoparticles measured at a temperature of $6 \mathrm{~K}$ and are associated with an asymmetric longitudinal $\mathrm{C}-\mathrm{H}\left(\mathrm{CH}_{2}\right)$ vibration.

The doping of $\mathrm{ZnO}$ with gallium displaces the short-wavelength transmission limit toward longer wavelengths and changes the shape of the transmittance curve in accordance with the varying Ga concentration [Figs. 4(a) and 4(b)]. Thus, if the transmittance limit of the ceramic into which 0.03 mass \% Ga was introduced (Table 1, 2a) is located in the 377-nm region, it lies in the 385-390-nm interval in samples that contain $0.05-0.1$ mass $\%$. The spectra of all the doped ceramics include maxima that shift from 565 to 485 or $465 \mathrm{~nm}$ as the gallium concentration increases from 0.03 to 0.1 mass $\%$. Since the sample with the minimum Ga concentration 
(a)

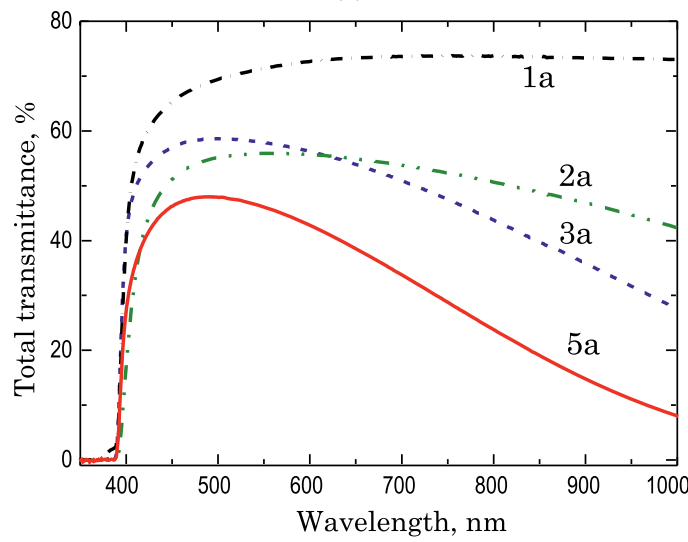

(c)

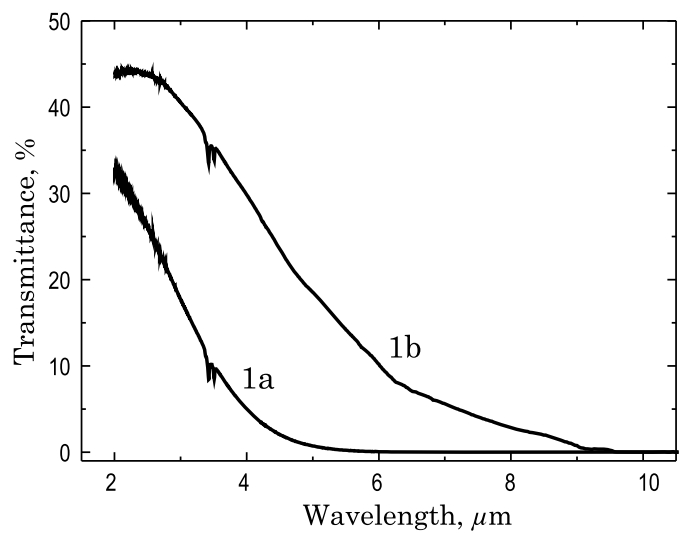

(b)

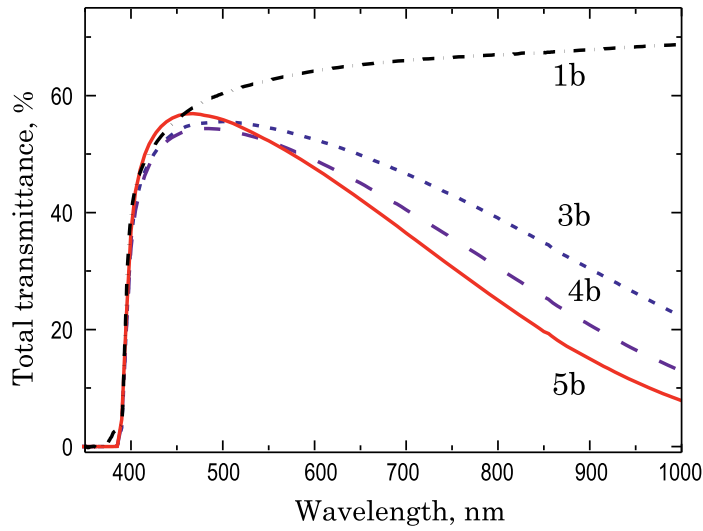

(d)

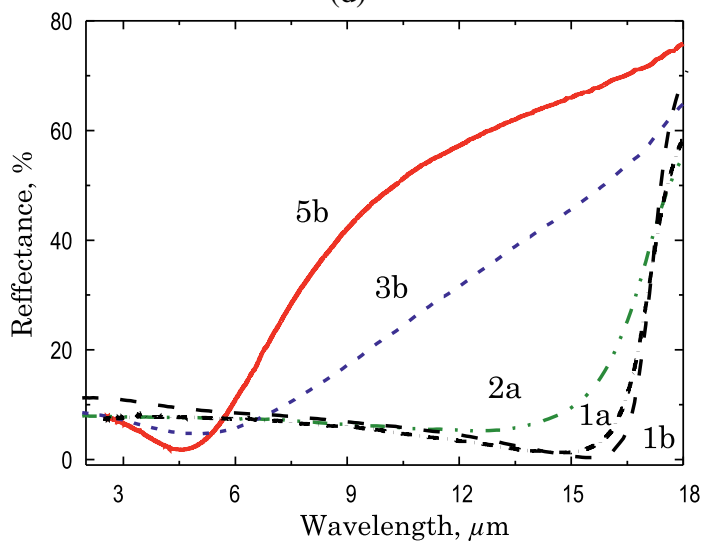

Fig. 4. Total transmission spectra of ceramic samples fabricated from TU-6-09-2175-77 and Sigma Aldrich powders (a, b), transmission spectrum (c), and reflection spectrum (d) in the mid-IR range of the $\mathrm{ZnO}$ and $\mathrm{ZnO}$ :Ga ceramics. The designations of the curves correspond to the sample numbers in Table 1.

(Table $1,2 \mathrm{a}$ ) is opaque for radiation with wavelength $2 \mu \mathrm{m}$, as established experimentally, it should be assumed that the longwavelength limit of all the doped ceramics lies in the region from 1 to $2 \mu \mathrm{m}$.

The observed absorption of the $\mathrm{ZnO}: \mathrm{Ga}$ ceramics in the visible and IR is free-charge-carrier absorption caused by the presence of shallow donors created by the gallium impurity.

The free-carrier concentration was determined from data obtained by studying the reflection spectra of $\mathrm{ZnO}$ and $\mathrm{ZnO}$ :Ga ceramics in the mid-IR [Fig. 4(d)]. The calculation used $[5,21]$

$$
n=\frac{\left(7.24 \times 10^{20}\right)}{\lambda_{\min }^{2}}
$$

where $\lambda_{\min }$ is the wavelength of the minimum in the IR reflection spectrum, expressed in micrometers, and $n$ is the freecarrier concentration $\left(\mathrm{cm}^{-3}\right)$.
The results of the calculation are shown in Table 2. The free-carrier concentration is somewhat higher in the $\mathrm{ZnO}$ ceramic fabricated from TU-6-09-2175-77 powder than in that made from Sigma Aldrich powder. An increase of the freecarrier concentration with an increase of the gallium content is observed in the doped ceramics, becoming virtually an order of magnitude higher than in the undoped ceramics.

The luminescence spectra of the ceramics measured in transmitted light is shown in Figs. 5(a)-5(d). The inset in Fig. 5(d) shows the luminescence spectra of the $\mathrm{ZnO}$ :Ga ceramics fabricated from the Sigma Aldrich powder, measured in reflected light. Undoped ceramics $1 \mathrm{a}$ and $1 \mathrm{~b}$ (Table 1) emit in the yellow-orange region and are characterized by a wide band with maximum in the $580-\mathrm{nm}$ region.

The radiation in the green-red region is usually associated with internal defects. Several luminescence bands are usually observed in various forms of $\mathrm{ZnO}$ : a band with maximum

Table 2. Wavelength of the Reflection-Spectrum Minimum and the Free-Carrier Concentration

\begin{tabular}{lccccc}
\hline Sample No. & $\mathbf{1 a}$ & $\mathbf{1 b}$ & $\mathbf{2 a}$ & $\mathbf{3 b}$ & $\mathbf{5 b}$ \\
\hline$\lambda_{\min }, \mu \mathrm{m}$ & 14.9 & 15.6 & 12.6 & 5 & 4.58 \\
$n, \mathrm{~cm}^{-3}$ & $3.26 \times 10^{18}$ & $2.975 \times 10^{18}$ & $4.56 \times 10^{18}$ & $2.896 \times 10^{19}$ & $3.44 \times 10^{19}$ \\
\hline
\end{tabular}


(a)

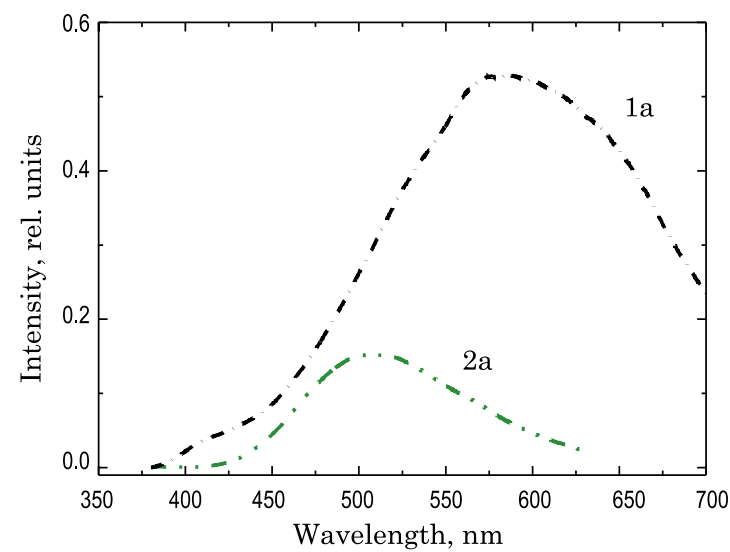

(c)

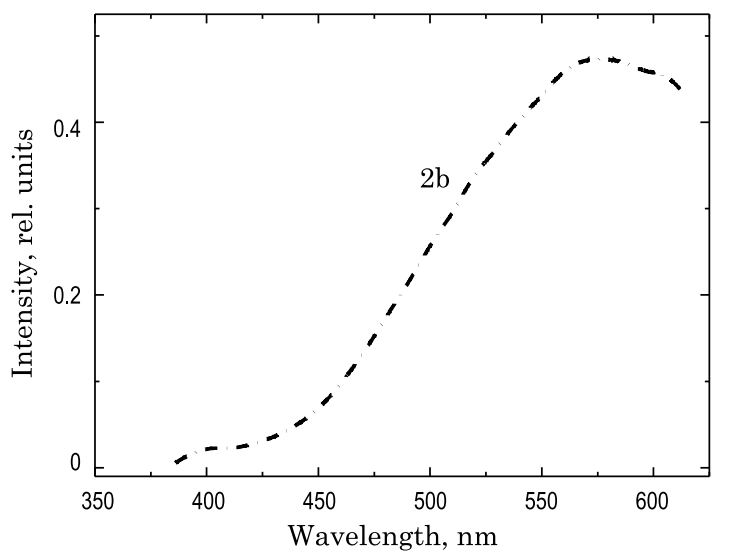

(b)

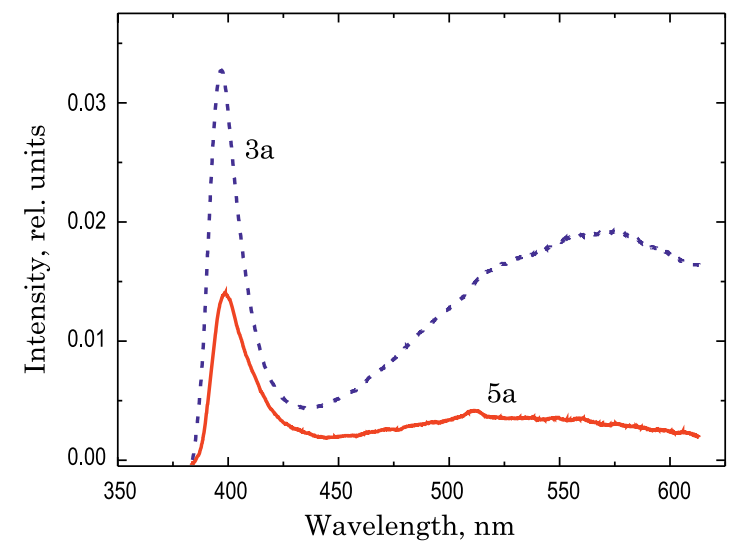

(d)

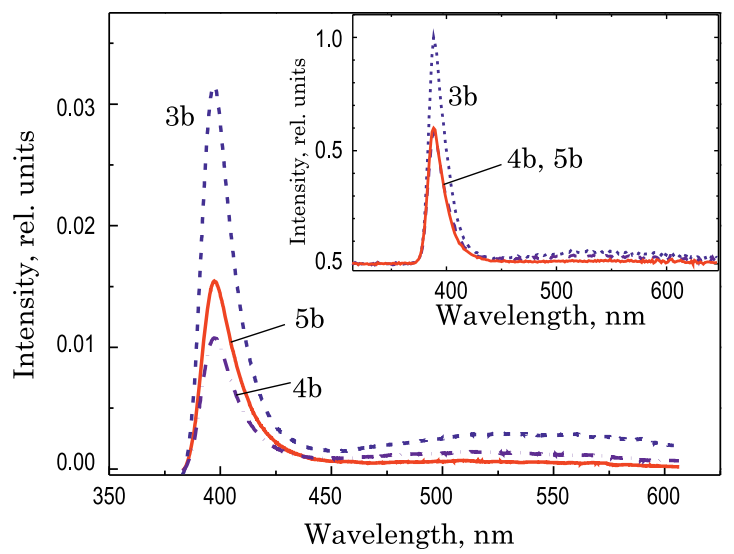

Fig. 5. X-ray luminescence spectra measured in transmitted light of $\mathrm{ZnO}$ and $\mathrm{ZnO}$ :Ga ceramics fabricated from (a), (b) TU-6-09-2175-77 and (c), (d) Sigma Aldrich powders. The inset in (d) is the X-ray luminescence spectra of ZnO:Ga samples measured in reflected light. The designations of the curves correspond to the sample numbers in Table 1 .

corresponding to $500 \mathrm{~nm}$, which characterizes samples obtained in reducing conditions, whereas samples obtained under oxidizing conditions are characterized by luminescence with a maximum corresponding to $600 \mathrm{~nm}[22,23]$. Some authors have expressed the assumption that oxygen and zinc vacancies are responsible for the luminescence bands with maxima corresponding to 500 and $600 \mathrm{~nm}$, respectively [22,23]. It is assumed in other papers that both luminescence bands are associated with modifications of oxygen vacancies: their various charge states $\left(V_{O}^{x / \bullet}\right.$ and $\left.V_{O}^{\bullet / \bullet \bullet}\right)$ [24], or their locatation either in the bulk or the near-surface layer of $\mathrm{ZnO}$ [25].

The exciton luminescence in $\mathrm{ZnO}$ ceramics is suppressed because the emission spectrum of the exciton luminescence overlaps the excitation spectra of the defect luminescence band, thereby reabsorbing the exciton luminescence and effectively transmitting energy from the excitons to the defect luminescence centers.

The introduction of 0.03 mass \% of gallium impurity into the $\mathrm{ZnO}$ (Table 1, 2a) broadens the LWL band, shifts the maximum into the green (to $510 \mathrm{~nm}$ ), and reduces the luminescence intensity by a factor of almost 3.5 by comparison with the undoped ceramic 1a [Table 1, Fig. 5(a)]. The exciton band in the spectrum of ceramic $2 \mathrm{a}$ (Table 1 ) that characterizes $\mathrm{ZnO}: \mathrm{Ga}$ is not observed.

The exciton band with maximum in the 398-nm region appears in ceramics $3 \mathrm{a}$ and $3 \mathrm{~b}$ (Table 1 ), doped with 0.05 mass $\%$ Ga [Figs. 5(b) and 5(d)]. A further increase of the gallium concentration reduces the intensity of the exciton peak as well as that of the defect band. It should be pointed out that the defect band in the doped ceramics fabricated from the TU-6-092175-77 powder made in Russia has higher intensity than do the samples produced with the Sigma Aldrich powder, where it is virtually suppressed. The spectra of the $\mathrm{ZnO}$ :Ga ceramics measured in reflected light [inset in Fig. 5(d)] differ from the spectra measured in transmitted light mainly by the position of the maximum of the exciton band, which lies in the 389-nm region. The obvious displacement of the edge-luminescence maximum by $10 \mathrm{~nm}$ toward longer wavelengths when it is measured in transmitted light is caused by the self-absorption of this radiation. A similar effect was noted in [21]. The high transmittance in the emission region of ceramic $5 \mathrm{~b}$ (Table 1 ) by comparison with sample $4 \mathrm{~b}$ (Table 1 ) is explained by its higher emission intensity when the measurement is made in transmitted light. 


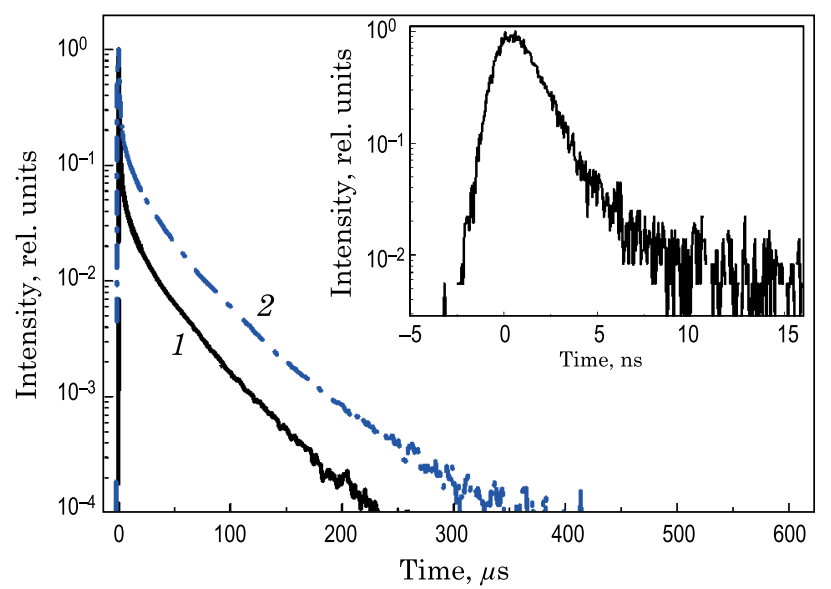

Fig. 6. Damping curves of X-ray luminescence excited by pulses of width 100 (1) and $1700 \mathrm{~ns}$ (2) in $\mathrm{ZnO}$ ceramic (sample 1b, Table 1). The inset shows the luminescence-damping kinetics of $\mathrm{ZnO}: \mathrm{Ga}$ ceramic (sample 5b, Table 1).

The collection of data obtained from sample $2 \mathrm{a}$ (containing 0.03 mass \% Ga, Table 1) concerning the lattice parameter $c$ [Fig. 1(a)], the grain size [Fig. 2(a)], the relatively low freecharge-carrier concentration, and the spectral content of the radiation by comparison with other doped ceramics make it realistic to assume that Ga may occupy an interstitial position in this case, since, in the words of the authors of [26], it is an inactive donor impurity.

The X-ray-luminescence damping curves that characterize undoped ceramics are shown in Fig. 6. In this figure, curves 1 and 2 are obtained when square excitation pulses 100 and 1700 ns wide, respectively, are used. The luminescencedamping curves have a complex, multicomponent character. Their shape can be approximated by the triple exponential falloff

$$
I=I_{1} \exp \left(-t / \tau_{1}\right)+I_{2} \exp \left(-t / \tau_{2}\right)+I_{3} \exp \left(-t / \tau_{3}\right),
$$

where $\tau_{i}$ is the damping time. The results of the approximation are shown in Table 3.

The observed complex shape of the damping curve is caused by two factors. The first one is explained by the fact that the spectra of the test ceramics with no dopants consist of several overlapping defect-luminescence bands. The luminescence band with maximum at about $500 \mathrm{~nm}$ in this case has more rapid luminescence damping than the band with maximum corresponding to $600 \mathrm{~nm}$ [27]. The second factor is the contribution of thermally delocalized charge carriers with shallow

Table 3. Approximation Parameters of the Luminescence-Damping Curves of ZnO Ceramic for a Triple-Exponential Falloff

\begin{tabular}{lccccccc}
\hline $\boldsymbol{t}_{\text {puls }}, \mathrm{ns}$ & $\boldsymbol{\tau}_{\mathbf{1}}, \boldsymbol{\mu s}$ & $\boldsymbol{L} \boldsymbol{Y}_{\mathbf{1}}$ & $\boldsymbol{\tau}_{\mathbf{2}}, \boldsymbol{\mu s}$ & $\boldsymbol{L} \boldsymbol{Y}_{\mathbf{2}}$ & $\boldsymbol{I}_{\mathbf{3}}$ & $\boldsymbol{\tau}_{\mathbf{3}}, \boldsymbol{\mu s}$ & $\boldsymbol{L} \boldsymbol{Y}_{\mathbf{3}}$ \\
\hline 1700 & 0.93 & 0.17 & 20 & 0.42 & 0.035 & 55 & 0.41 \\
100 & 0.54 & 0.27 & 10 & 0.27 & 0.02 & 42 & 0.46 \\
\hline
\end{tabular}

Note. $t_{\text {puls }}$ is the excitation pulse width, and $L Y_{i}$ is the normalized light output of the component with damping time $\tau_{i}, L Y_{i}=\left(I_{i} \tau_{i}\right) /\left(I_{1} \tau_{1}+I_{2} \tau_{2}+I_{3} \tau_{3}\right)$. traps. As can be seen from Fig. 6 and Table 3, increasing the width of the exciting pulse increases the damping constant of the luminescence kinetics, and this is characteristic of emission processes in which charge traps actively participate. The presence of several forms of charge traps with various parameters and their interaction with each other significantly complicates the problem of mathematically describing the process and results in a multicomponent luminescence-damping curve.

Gallium-doped ceramics in which the defect luminescence is suppressed possess kinetic damping with time constants of 1.1 and $10 \mathrm{~ns}$ (inset in Fig. 6). The damping time of $1.1 \mathrm{~ns}$ corresponds to typical exciton lifetimes in $\mathrm{ZnO}$, measured with photoexcitation [1]. The component with a damping constant of $10 \mathrm{~ns}$ probably results from incompletely quenched defect luminescence.

Annealing in an argon-hydrogen atmosphere is used to increase the efficiency of exciton luminescence and to suppress the defect-emission band in powders, films, and nanoparticles $[3,28,29]$. A mixture that contains $5 \% \mathrm{H}_{2}$ is most often used. It is assumed that the hydrogen plays a double role: It eliminates an excess of oxygen and reduces the number of zinc vacancies that form the shallow acceptor $\mathrm{H}_{\mathrm{Zn}}^{\prime}$. The mechanism proposed in the literature for increasing the edge-luminescence intensity with argon-hydrogen heat treatment consists of recombining the electrons localized on the $\mathrm{Ga}^{3+}$ donor level with the holes trapped by the shallow acceptor $\mathrm{H}_{\mathrm{Zn}}^{\prime}$ [3].

Our experiments on the heat treatment of $\mathrm{ZnO}$ : Ga ceramics for $120 \mathrm{~min}$ in an atmosphere that contains $5 \%$ hydrogen at a temperature of $600^{\circ} \mathrm{C}$ allowed us to increase the exciton luminescence intensity by a factor of 3 on average. However, this effect was observed in a layer about $100 \mu \mathrm{m}$ thick [30]. Our latest experiments that involve varying the annealing parameters show that the exciton-luminescence intensity can be increased more substantially. It should be pointed out that, even though the luminescence intensity is increased, heat treatment in an argon-hydrogen gas mixture does not affect the luminescence kinetics of either doped or undoped ceramics.

Samples of $\mathrm{ZnO}$ :Ga ceramics were tested using heavy-ion beams at GSI (Helmholtzzentrum für Schwerionenforschung GmbH, Germany) [31]. As shown by the studies, their temporal characteristics approach those of a VS-400 plastic scintillator, while the radiation stability is more than an order of magnitude greater than that of the latter and is comparable with silicon and diamond detectors. Thus, $\mathrm{ZnO}$ :Ga ceramics can be an effective replacement of the plastic scintillators being used.

\section{CONCLUSION}

The grains of $\mathrm{ZnO}$ and $\mathrm{ZnO}: \mathrm{Ga}$ ceramics become predominantly oriented along the prism planes during hot pressing, and this ensures that the $\mathrm{ZnO}$ samples have relatively high transparency in the visible region.

The introduction of from 0.03 to 0.1 mass \% Ga into the $\mathrm{ZnO}$ structure reduces the grain size.

It has been established that different starting powders have an effect on the free-carrier concentration in the $\mathrm{ZnO}$ ceramic and its long-wavelength transmission limit, as well as on the intensity of the long-wavelength luminescence band in the $\mathrm{ZnO}: \mathrm{Ga}$ ceramic. 
The maximum exciton-luminescence intensity in the $\mathrm{ZnO}: \mathrm{Ga}$ ceramic is observed when the $\mathrm{Ga}$ concentration is 0.05 mass $\%$.

It has been established that it is promising to use $\mathrm{ZnO}$ : $\mathrm{Ga}$ as a scintillation ceramic for recording heavy ions. It possesses an exciton-luminescence damping time of about $1.1 \mathrm{~ns}$ and high radiation stability.

Funding. Russian Foundation for Basic Research (RFBR) (18-52-76002).

\section{REFERENCES}

1. U. Orgur, Ya. I. Alivov, C. Liu, A. Teke, M. A. Reshnikov, S. Dogan, V. Avrutin, S.-J. Cho, and H. Morkoc, "A comprehensive review of ZnO materials and devices," J. Appl. Phys. 98, 041301 (2005).

2. T. Makino, Y. Segawa, S. Yoshida, A. Tsukazak, A. Ohtomo, and M. Kawasaki, "Gallium concentration dependence of room-temperature near-band-edge luminescence in n-type ZnO:Ga," Appl. Phys. Lett. 85, 759-761 (2004).

3. E. D. Bourret-Courchesne, S. E. Derenzo, and M. J. Weber, "Development of $\mathrm{ZnO}: \mathrm{Ga}$ as an ultra-fast scintillator," Nucl. Instrum. Methods Phys. Res. A 601, 358-363 (2009).

4. J. C. Cooper, D. S. Koltick, J. T. Mihalzo, and J. S. Neal, "Evaluation of $\mathrm{ZnO}(\mathrm{Ga})$ coatings as alpha particle transducers within a neutron generator," Nucl. Instrum. Methods Phys. Res. A 505(1-2), 498501 (2003).

5. J. S. Neal, N. C. Giles, X. Yang, R. A. Wall, K. B. Ucer, R. T. Williams, D. J. Wisniewski, L. A. Boatner, V. Rengarajan, and B. Nemeth, "Evaluation of melt-grown, $\mathrm{ZnO}$ single crystals for use as alphaparticle detectors," IEEE Trans. Nucl. Sci. 55, 1397-1403 (2008).

6. E. I. Gorokhova, G. V. Anan'eva, V. A. Demidenko, P. A. Rodnyi, I. V. Khodyuk, and E. D. Bourret-Courchesne, "Optical, luminescence, and scintillation properties of $\mathrm{ZnO}$ and $\mathrm{ZnO}$ :Ga ceramics," J. Opt. Technol. 75(11), 741-746 (2008) [Opt. Zh. 75(11), 66-72 (2008)].

7. J. S. Neal, D. M. DeVito, B. L. Armstrong, M. Hong, B. Kesanli, X. Yang, N. C. Giles, J. Y. Howe, J. O. Ramey, D. J. Wisniewski, M. Wisniewska, Z. A. Munir, and L. A. Boatner, "Investigation of ZnO-based polycrystalline ceramic scintillators for use as $\alpha$-particle detectors," IEEE Trans. Nucl. Sci. 56, 892-898 (2009).

8. K. A. Chernenko, E. I. Gorokhova, S. B. Eron'ko, A. V. Sandulenko, I. D. Venevtsev, H. Wieczorek, and P. A. Rodnyi, "Structural, optical and luminescent properties of $\mathrm{ZnO}: \mathrm{Ga}$ and $\mathrm{ZnO}: \mathrm{In}$ ceramics," IEEE Trans. Nucl. Sci. 65(8), 2196-2202 (2018).

9. J. A. R. Márquez, C. M. B. Rodríguez, C. M. Herrera, E. R. Rosas, O. Z. Angel, and O. T. Pozos, "Effect of surface morphology of $\mathrm{ZnO}$ electrodeposited on photocatalytic oxidation of methylene blue dye. Part I: Analytical study," Int. J. Electrochem. Sci. 6, 4059-4069 (2011).

10. K. A. Chernenko, S. B. Mikhrin, H. Wieczorek, C. R. Ronda, and P. A. Rodnyı̆, "Source of rectangular X-ray pulses for studying scintillators," Tech. Phys. Lett. 41(10), 971-973 (2015) [Pis'ma Zh. Tekh. Fiz. 41(20), 1-7 (2015)].

11. P. A. Rodnyi, S. B. Mikhrin, A. N. Mishin, and A. V. Sidorenko, "Small-size pulsed X-ray source for measurements of scintillator decay time constants," IEEE Trans. Nucl. Sci. 48(6), 2340-2343 (2001).

12. A. R. Kaul, O. Yu. Gorbenko, A. N. Botev, and L. I. Burova, "MOCVD of pure and Ga-doped epitaxial ZnO," Superlattices Microstruct. 38, 272-282 (2005).

13. S. Chen, G. Carraro, D. Barreca, A. Sapelkin, W. Chen, X. Huang, Q. Cheng, F. Zhange, and R. Binions, "Aerosol-assisted chemical vapour deposition of Ga-doped ZnO films for energy efficient glazing: effects of doping concentration on the film-growth behaviour and optoelectronic properties," J. Mater. Chem. A 3, 13039-13049 (2015).

14. R. D. Shannon, "Revised effective ionic radii and systematic studies of interatomic distances in halides and chalcogenides," Acta Crystallogr. A 32, 751-767 (1976).

15. E. I. Gorokhova, S. B. Eron'ko, A. M. Kul'kov, E. A. Oreshchenko, K. L. Simonova, K. A. Chernenko, I. D. Venevtsev, P. A. RodnyĬ, K. P. Lott, and $\mathrm{H}$. Wieczorek, "Development and study of $\mathrm{ZnO}$ : in optical scintillation ceramic," J. Opt. Technol. 82(12), 837-842 (2015) [Opt. Zh. 82(12), 78-85 (2015)].

16. S. S. Gorelik, Recrystallization of Metals and Alloys (Metallurgiya, Moscow, 1978).

17. N. A. Vorob'eva, "Nanocrystalline $\mathrm{ZnO}(\mathrm{M})(\mathrm{M}=\mathrm{Ga}$, In) for gas sensors and transparent electrodes," Candidate for Chemical Sciences Dissertation (Moscow State University, 2015).

18. E. V. Kortunova, N. G. Nikolaeva, P. P. Chvanski, V. V. Maltsev, E. A. Volkova, E. V. Koporulina, N. I. Leonyuk, and T. F. Kuech, "Hydrothermal synthesis of improved $\mathrm{ZnO}$ crystals for epitaxial growth of GaN thin films," J. Mater. Sci. 43, 2336-2341 (2008).

19. I. P. Kuz'mina and V. P. Nikitenko, Zinc Oxide-Production and Optical Properties (Nauka, Moscow, 1984).

20. P. Saadatkia, G. Ariyawansa, K. D. Leedy, D. C. Look, L. A. Boatner, and F. A. Selim, "Fourier transform infrared spectroscopy measurements of multiphonon and free-carrier absorption in $\mathrm{ZnO}$," J. Electronic Mat. 45(12), 6329-6336 (2016).

21. X. Yang, "Electrical and optical properties of zinc oxide for scintillator applications," Dissertation for degree D.Ph. in Physics (West Virginia University, Morgantown, WV, 2008).

22. T. M. Borseth, B. G. Svensson, and A. Yu. Kuznetsov, "Identification of oxygen and zinc vacancy optical signals in $\mathrm{ZnO}$," Appl. Phys. Lett. 89, 262112 (2006).

23. C. Ton-That, L. Weston, and M. R. Phillips, "Characteristics of point defects in the green luminescence from $\mathrm{Zn}$ and O-rich $\mathrm{ZnO}$," Phys. Rev. B 86, 115205 (2012).

24. J. D. Ye, S. L. Gu, F. Qin, S. M. Zhu, S. M. Liu, X. Zhou, W. Liu, L. Q. Hu, R. Zhang, Y. Shi, and Y. D. Zheng, "Correlation between green luminescence and morphology evolution of $\mathrm{ZnO}$ films," Appl. Phys. A 81, 759-762 (2005).

25. Y. Y. Tay, T. T. Tan, F. Boey, M. H. Liang, J. Ye, Y. Zhao, T. Norby, and $\mathrm{S}$. Li, "Correlation between the characteristic green emissions and specific defects of ZnO," Phys. Chem. Chem. Phys. 12, 23732379 (2010).

26. V. Khranovskyy, U. Grossner, V. Lazorenko, G. Lashkarev, B. G. Svensson, and R. Yakimova, "Conductivity increase of $\mathrm{ZnO}: \mathrm{Ga}$ films by rapid thermal annealing," Superlattices Microstruct. 42, 379-386 (2007).

27. K. A. Chernenko, O. G. Klimova, E. I. Gorokhova, G. G. Klimov, A. V. Semencha, and P. A. Rodnyi, "The effect of annealing on spectra and decay time of X-ray luminescence of zinc oxide powders," IOP Conf. Ser.: Mater. Sci. Eng. 49, 012028 (2013).

28. Q. Li, X. Liu, M. Gu, S. Huang, J. Zhang, C. Ni, Y. Hu, Q. Wu, and $\mathrm{S}$. Zhao, "X-ray excited luminescence of $\mathrm{Ga}$ - and In-doped $\mathrm{ZnO}$ microrods by annealing treatment," Superlattices Microstruct. 98, 351-358 (2016).

29. Q. Li, X. Liu, M. Gu, F. Li, J. Zhang, Q. Wu, S. Huang, and S. Liu, "Large enhancement of X-ray excited luminescence in Ga-doped ZnO-nanorod arrays by hydrogen annealing," Appl. Surf. Sci. 433 , 815-820 (2018).

30. P. Rodnyi, K. Chernenko, O. Klimova, V. Galkin, A. Makeenko, E. Gorokhova, D. Buettner, W. Keur, and H. Wieczorek, "Influence of annealing on the scintillation properties of zinc oxide powders and ceramics," Radiat. Meas. 90, 136-139 (2016).

31. P. Boutachov, A. Reiter, B. Walasek-Hohne, E. Gorokhova, and $\mathrm{P}$. Rodnyi, "Radiation hardness investigation of $\mathrm{ZnO}(\mathrm{Ga})$ and $\mathrm{ZnO}(\mathrm{In})$ with heavy ion beams," in Abstracts of SCINT 2017 14th International Conference on Scintillating Materials and Their Applications, 2017. 